\title{
Intravenous therapy
}

\author{
Authors: Syed Haydar, Frances Asota and Mary McGinty
}

\section{Aims}

The majority of patients admitted to hospital will become a recipient of an intravenous cannula (IVC) during their inpatient admission. Numerous case reports have demonstrated adverse events and critical incidents as a result of accidental intraarterial (IA) cannulation, specifically in the antecubital fossa (ACF). ACF cannulation increases the risk of extravasation injury, phlebitis and accidental IA insertion. The Royal College of Nursing (RCN) and local trust guidelines recommend that insertion site should be chosen in the following order: hand, wrist, forearm, ACF veins. Sites over articulating joints should also be avoided. Therefore, the IV Therapy Group conducted a snapshot audit to assess all patients with IVC. The aim of this project was to identify and investigate the reasons for increased ACF cannulation after an assessment of vascular integrity, and to highlight the extent of this issue in choice of IVC siting.

\section{Methods}

Patients with IVC in situ were reviewed on all inpatient wards at Colchester General Hospital (except EAU and A\&E) using a pro forma. Data collection was performed by the IV Therapy Group on 2 May 2013. Data were collected from patients, medical notes, nursing records, medication charts, and then audited against trust guidelines for IVC insertion.

\section{Results}

In the 161 patients that were reviewed, we demonstrated that position of IVC was distributed almost evenly between the hand (35\%), forearm and wrist (38\%), and ACF (27\% deemed significantly high by the IV Therapy Group -of which only $30 \%$ had poor vascular integrity). Insertion details were not documented in $81 \%$ of cases. Training issues were identified amongst all staff groups, and human factors in technique and beliefs were addressed. We established re-education for staff members who cannulate in the form of workshops at trust inductions. We designed a poster and screensaver for all inpatient wards and trust computers respectively - which included 'The 4-point check for IV cannulation', trust guidelines, and a list of warning signs for accidental IA cannulation to raise awareness and implement a change in current IV cannulation technique.

Authors: Colchester Hospital University NHS Foundation Trust Colchester, UK

\section{Conclusions}

For patient safety, we advocate against routine ACF cannulation, as it risks the potential for accidental IA insertion, and the subsequent harm caused by unintentional IA drug administration. This can be prevented by assessing vascular integrity, starting distally when attempting intravenous cannulation, and recognising when a cannula is unintentionally inserted into an artery.

\section{Conflict of interest statement}

None declared. 